\title{
Alpha-enolase in viral target cells suppresses the human immunodeficiency virus type 1 integration
}

\author{
Naoki Kishimoto ${ }^{1}$, Kengo Yamamoto ${ }^{1}$, Nozomi Iga ${ }^{1}$, Chie Kirihara ${ }^{1}$, Towa Abe ${ }^{1}$, Nobutoki Takamune ${ }^{2}$ \\ and Shogo Misumi ${ }^{1^{*}}$ (D)
}

\begin{abstract}
Background: A protein exhibiting more than one biochemical function is termed a moonlighting protein. Glycolytic enzymes are typical moonlighting proteins, and these enzymes control the infection of various viruses. Previously, we reported that glyceraldehyde 3-phosphate dehydrogenase (GAPDH) and alpha-enolase (ENO1) are incorporated into human immunodeficiency virus type 1 (HIV-1) particles from viral producer cells and suppress viral reverse transcription independently each other. However, it remains unclear whether these proteins expressed in viral target cells affect the early phase of HIV-1 replication.
\end{abstract}

Results: Here we show that the GAPDH expression level in viral target cells does not affect the early phase of HIV-1 replication, but ENO1 has a capacity to suppress viral integration in viral target cells. In contrast to GAPDH, suppression of ENO1 expression by RNA interference in the target cells increased viral infectivity, but had no effect on the expression levels of the HIV-1 receptors CD4, CCR5 and CXCR4 and on the level of HIV-1 entry. Quantitative analysis of HIV-1 reverse transcription products showed that the number of copies of the late products (R/gag) and two-longterminal-repeat circular forms of viral cDNAs did not change but that of the integrated (Alu-gag) form increased. In contrast, overexpression of ENO1 in viral target cells decreased viral infectivity owing to the low viral integration efficiency. Results of subcellular fractionation experiments suggest that the HIV integration at the nucleus was negatively regulated by ENO1 localized in the nucleus. In addition, the overexpression of ENO1 in both viral producer cells and target cells most markedly suppressed the viral replication.

Conclusions: These results indicate that ENO1 in the viral target cells prevents HIV-1 integration. Importantly, ENO1, but not GAPDH, has the bifunctional inhibitory activity against HIV-1 replication. The results provide and new insights into the function of ENO1 as a moonlighting protein in HIV-1 infection.

Keywords: Human immunodeficiency virus type 1, Alpha-enolase, Reverse transcription, Integration

\footnotetext{
*Correspondence: misumi@gpo.kumamoto-u.ac.jp

${ }^{1}$ Department of Environmental and Molecular Health Sciences,

Faculty of Medical and Pharmaceutical Sciences, Kumamoto University, Kumamoto 862-0973, Japan

Full list of author information is available at the end of the article
}

\begin{abstract}
Background
Human immunodeficiency virus type 1 (HIV-1) replication depends on not only viral proteins, but also host proteins. Several studies demonstrated that host proteins play a critical role in HIV-1 replication with positive or negative regulation. For example, CD4, CCR5 and/or CXCR4 are required in the viral entry step by binding with viral envelope proteins, and lens epithelium-derived growth factor (LEDGF/p75) is utilized as
\end{abstract}

c) The Author(s) 2020. This article is licensed under a Creative Commons Attribution 4.0 International License, which permits use, sharing, adaptation, distribution and reproduction in any medium or format, as long as you give appropriate credit to the original author(s) and the source, provide a link to the Creative Commons licence, and indicate if changes were made. The images or other third party material in this article are included in the article's Creative Commons licence, unless indicated otherwise in a credit line to the material. If material is not included in the article's Creative Commons licence and your intended use is not permitted by statutory regulation or exceeds the permitted use, you will need to obtain permission directly from the copyright holder. To view a copy of this licence, visit http://creativeco mmons.org/licenses/by/4.0/. The Creative Commons Public Domain Dedication waiver (http://creativecommons.org/publicdomain/ zero/1.0/) applies to the data made available in this article, unless otherwise stated in a credit line to the data. 
a cofactor of HIV-1 integrase during integration [1-8]. In contrast, host proteins, such as SAM domain and HD domain-containing protein 1 and apolipoprotein $B$ mRNA-editing enzyme-catalytic polypeptide-like-3G, suppress HIV-1 replication in the early phase by degrading dNTPs and inducing G-to-A hypermutation in the viral genome, respectively [9-12]. Therefore, identification of the host proteins involved in HIV-1 replication is one way to understand their regulatory mechanism in HIV replication.

More than 300 proteins including glycolytic enzymes such as glyceraldehyde 3-phosphate dehydrogenase (GAPDH) and alpha-enolase (ENO1) have been identified as moonlighting proteins that perform more than one function [13-15]. Their multiple functions are not due to splicing variants, protein isoforms, or co-/post-translational modifications but to their diversity as receptors, scaffolds, enzymes, chaperones or transcription factors. Interestingly, GAPDH is an archetypal moonlighting protein that is involved in glycolysis, the carbon reduction cycle, the exportation of nuclear RNA, DNA repair and apoptosis [15-18]. Furthermore, GAPDH binds to viral RNA, such as the hepatitis A virus, hepatitis $C$ virus and human parainfluenza virus, to regulate viral replication [19-21]. In addition, ENO1, which was initially identified as a glycolytic enzyme that catalyzes the conversion of 2-phosphoglycerate to phosphoenolpyruvate in glycolysis, is also a moonlighting protein functioning as a plasminogen receptor, a heat-shock protein and a hypoxic stress protein, and associates with infectious viruses such as the Sendai and dengue viruses [22, 23]. These studies raised the question of whether glycolytic enzymes can regulate HIV-1 replication as moonlighting proteins. We previously demonstrated that GAPDH binds to HIV-1 precursor proteins and is incorporated into virions to prevent the incorporation of the tRNA ${ }^{\text {Lys3 }}$ complex and inhibits the initiation of HIV-1 reverse transcription [24, 25]. Moreover, we found that packaging of ENO1 into viral particles affects the early stage of viral reverse transcription [26]. However, the effects of the moonlighting functions of GAPDH and ENO1 on HIV-1 target cells have not been clarified.

In this study, we found that GAPDH expressed in HIV-1 target cells does not affect HIV-1 replication, but ENO1 expressed in HIV-1 target cells has the ability to decrease the efficiency of virus integration without altering the reverse transcription efficiency. Considering that ENO1 incorporated into virions suppresses the early stage of reverse transcription, it is reasonable to hypothesize that ENO1 has bifunctional inhibitory activity against HIV-1 replication. These findings provide a novel regulatory mechanism of HIV-1 replication by host proteins.

\section{Results}

\section{ENO1 in HIV-1 target cells influences viral infectivity}

We previously reported that GAPDH and ENO1 are incorporated into HIV-1 particles, and virion-incorporated GAPDH and ENO1 inhibit viral reverse transcription independently of each other [24, 26]. Therefore, in this study, we examined whether GAPDH and ENO1 expression levels were changed by HIV-1 infection. Cell lysates derived from a T-cell line, CEM cells, and a chronically HIV-1-infected T-cell line, CEM/LAV-1 cells, were subjected to western immunoblotting. As a result, we detected no significant difference in the expression level of GAPDH between CEM and CEM/LAV-1 cells (Fig. 1a). In addition, GAPDH knockdown in TZM-bl cells maintained comparable levels of HIV-1 infectivity (Fig. 1b, c). On the other hand, the expression level of ENO1 was lower in CEM/LAV-1 cells than in CEM cells (Fig. 1d), and ENO1 knockdown in TZM-bl cells increased HIV-1 infectivity (Fig. 1e, f) without affecting cell viability (Fig. 1g). These findings suggest that ENO1 may have higher inhibitory activity against HIV-1 infection than GAPDH in target cells, and ENO1 in viral target cells may inhibit HIV-1 replication via a mechanism similar to or different from that underlying the inhibitory activity of virion-packaged ENO1.

\section{ENO1 knockdown in HIV-1 target cells increases viral integration efficiency}

To gain further insight into the function of ENO1 in HIV-1 target cells, we examined postentry steps in ENO1 knockdown cells. As shown in Fig. 2a, flow cytometry showed that treatment of TZM-bl cells with ENO1-specific siRNA had no effect on surface expression levels of the HIV-1 receptors CD4, CCR5 and CXCR4. In addition, measurement of cytosolic p24 isolated from HIV-1-infected TZM-bl cells by a previously described method [27] demonstrated that HIV-1 was able to penetrate into target cells regardless of the ENO1 expression level in the cells (Fig. 2b). We next investigated whether ENO1 knockdown in TZM-bl cells enhances the viral reverse transcription because low-level-ENO1-packaging virus, which was prepared by transfection of CEM/LAV-1 cells with an ENO1specific siRNA, showed an increased reverse transcription efficiency [26]. Unexpectedly, ENO1 knockdown has no effect on the abundance of late R/gag products of reverse transcription (Fig. 2c). Furthermore, the number of copies of two-long-terminal-repeat (2-LTR) circular DNA products, which are generally used as a marker of viral cDNA nuclear import, also showed no significant difference between ENO1-knockdown cells and control cells (Fig. 2d). However, when we performed nested Alu-gag PCR analysis, which is 
a

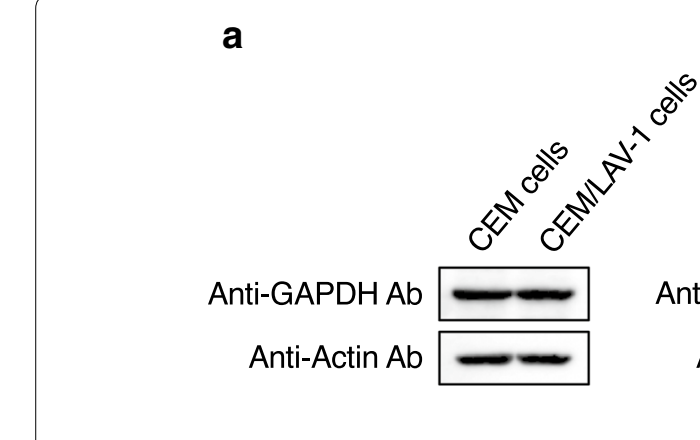

b

ti-GAPDH Ab

Anti-Actin Ab

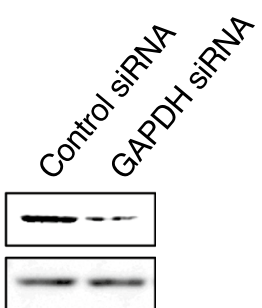

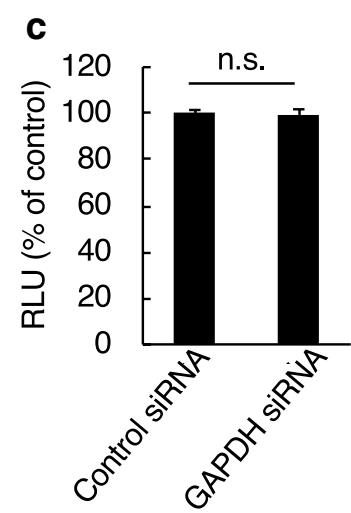

e

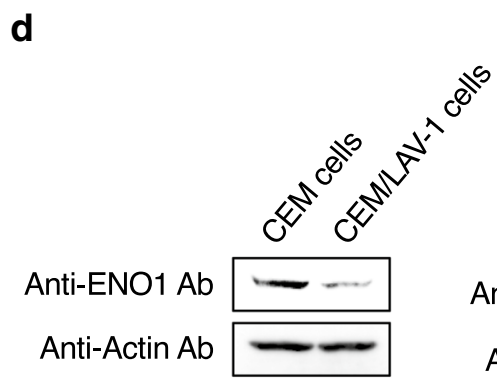

Anti-ENO1 Ab

Anti-Actin Ab

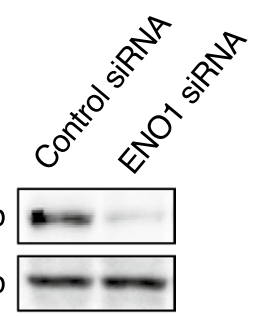

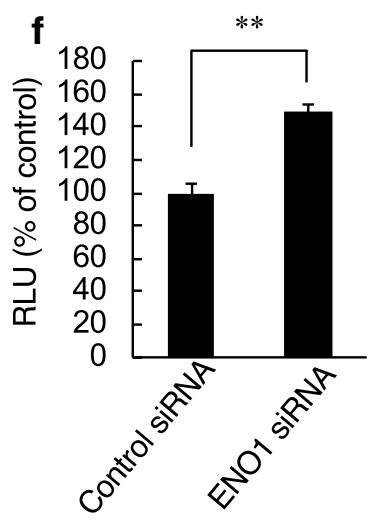

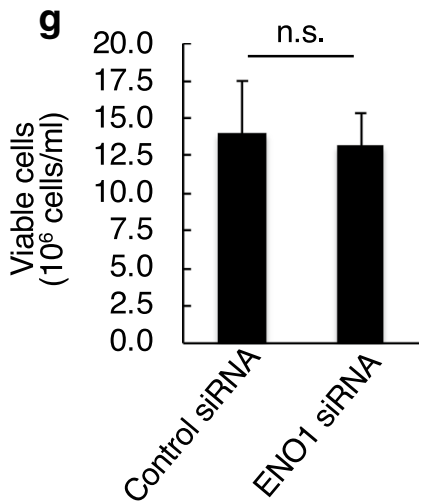

Fig. 1 ENO1, but not GAPDH, in target cells affects HIV-1 replication. a Western immunoblotting monitoring of GAPDH expression in either CEM

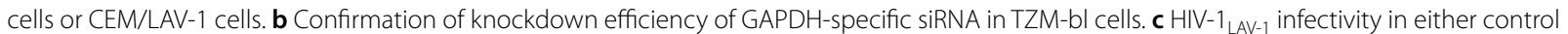
or GAPDH-knockdown TZM-bl cells. The value in the control-siRNA-treated TZM-bl cells was set as 100\%. d Western immunoblotting monitoring of ENO1 expression in either CEM cells or CEM/LAV-1 cells. e Confirmation of knockdown efficiency of ENO1-specific siRNA in TZM-bl cells. $\mathbf{f}$ HIV-1 LAV-1 $_{\text {L }}$ infectivity in ENO1-knockdown TZM-bl cells. The infectivity in the control-siRNA-treated TZM-bl cells was set as 100\%. $\mathbf{g}$ Effects of ENO1 siRNA treatment on viability of TZM-bl cells. Viable cells were evaluated by trypan blue staining. The infectivity was examined at 2 days postinfection with HIV-1 LAV $-1_{1}$ and assessed on the basis of the luciferase activity in lysates of any siRNA-treated TZM-bl cells. Data are mean values \pm SE from triplicate tests. The significance of difference (Student's t-test) is indicated as follows: ${ }^{* *}, p<0.01$; n.S., not significant

generally used for calculation of integrated viral cDNA, we found that ENO1 knockdown increased the integration efficiency, which correlates with an enhanced
HIV-1 infection (Fig. 2e). These findings indicate that unlike the inhibitory effect of virion-packaged ENO1, 

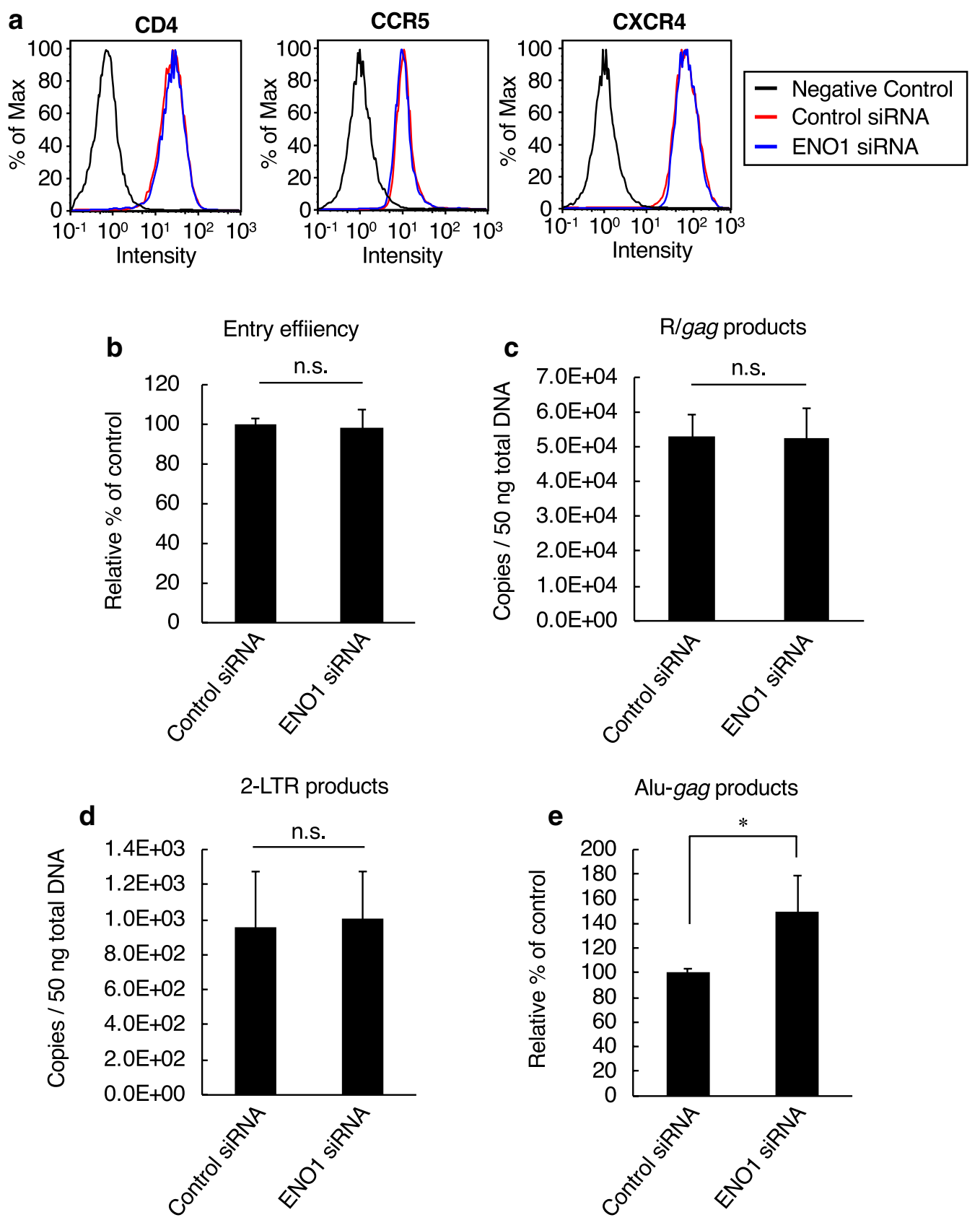

Fig. 2 Effects of ENO1 knockdown in HIV-1 target cells on postentry step. a Flow cytometry monitoring of HIV-1 receptor CD4 and coreceptors CCR5 and CXCR4. Cell stained with isotype control antibody are indicated as negative control (black line). The expression levels of CD4, CCR5 and CXCR4 in control-siRNA (red line) or ENO1-specific-siRNA (blue line) -treated TZM-bl are shown. The expression level of each protein was examined just before the cells were infected with the virus. $\mathbf{b}$ Entry efficiency of HIV-1 $1_{\text {LAV-1 }}$ in either control or ENO1-knockdown TZM-bl cells. Entry efficiency was assessed on the basis of the amount of p24 from the cytosolic fraction of TZM-bl cells. The entry efficiency in the control-siRNA-treated TZM-bl cells was set as 100\%. c Effect of ENO1 knockdown in TZM-bl cells on viral reverse transcription. The amount of R/gag products of viral reverse transcription was determined by quantitative real-time PCR analysis. $\mathbf{d}$ Effect of ENO1 knockdown in TZM-bl cells on viral cDNA nuclear import. The amount of 2-LTR circle products was determined by quantitative real-time PCR analysis. e Integration efficiency of HIV-1 LAV-1 $_{\text {in either }}$ control or ENO1-knockdown TZM-bl cells. Relative amount of Alu-gag products was determined by nested-PCR. The integration efficiency in the control-siRNA-treated TZM-bl cells was set as 100\%. Data are mean values \pm SE from triplicate tests. The significance of difference (Student's t-test) is indicated as follows: ${ }^{*}, p<0.05 ;$ n.S., not significant 
ENO1 in viral target cells inhibits HIV replication by preventing HIV-1 integration.

\section{ENO1 overexpression in HIV-1 target cells decreases viral integration efficiency}

Because ENO1 knockdown in the viral target cells resulted in increased HIV-1 infectivity, we next investigated the effect of ENO1 overexpression. To overexpress ENO1 in viral target cells, TZM-bl cells were transfected with an ENO1-V5 expression vector (Fig. 3a). As a result, ENO1 overexpression in HIV-1 target cell decreased viral integration efficiency (Fig. 3b). This result indicated that ENO1 impaired HIV-1 infection in viral target cells. To further examine whether ENO1 overexpression affects CD4, CXCR4 and CCR5 expression levels, we performed flow cytometry using anti-CD4, anti-CCR5 and anti-CXCR4 antibodies. The results showed that ENO1 overexpression had no effect on the expression levels of HIV-1 receptors, indicating that the viral entry step was unaffected by ENO1 overexpression (Fig. 3c). In addition, quantitative real-time PCR showed that ENO1 overexpression also had no effect on the number of copies of late R/gag (Fig. 3d) and 2-LTR circular DNA products (Fig. 3e). However, as expected, nested Alu-gag PCR analysis showed that ENO1 overexpression decreased viral integration efficiency compared with control vector treatment (Fig. 3f). Previously, we reported that highlevel-ENO1-packaging virus, which was prepared by cotransfection of HEK293 cells with pNL-CH and ENO1V5 expression vector, showed decreased number of copies of viral reverse transcription products [26]. Therefore, on the basis of these findings, we hypothesized that ENO1 is a bifunctional inhibitory protein that inhibits reverse transcription and integration processes.

\section{Nuclear ENO1 prevents HIV-1 integration}

To prevent HIV-1 integration, ENO1 should be in the viral target cell nucleus. Therefore, we confirmed the subcellular localization of ENO1. Endogenous ENO1 in TZM-bl cells was stained with specific antibodies and detected by fluorescence microscopy. We observed a weak nuclear ENO1-specific signal (Fig. 4a, left top panel). Interestingly, a clearer signal from V5-tagged ENO1, which was expressed by transfection in TZM-bl cells, indicated that ENO1 was present in the nucleus (Fig. 4a, right top panel). To clarify the ENO1 localization in more detail, we next fractionated the cells and detected endogenous ENO1, ENO1-V5, lactate dehydrogenase (LDH) and histone deacetylase1 (HDAC1) by western immunoblotting. LDH and HDAC1 were detected as a cytosolic marker and a nuclear marker, respectively. As shown in Fig. 4b, large amounts of ENO1 and ENO1-V5 were detected in the cytosolic fraction. Interestingly, small amounts of ENO1 and ENO1-V5 were also detected in the nuclear fraction, suggesting that the suppression of HIV infection by ENO1 overexpression (Fig. 3f) depended on V5-tagged ENO1 translocated into the nucleus. To eliminate the effects of the V5-tag, treatment of the untagged ENO1 expression vector increased the amount of ENO1 in the nucleus (Additional file 1: Figure S1A) and enhanced the inhibitory effect of ENO1 (Additional file 1: Figure S1B). Next, since TZM-bl cells are derived from HeLa cells, not immune cells, we also determined whether a small amount of ENO1 is present in the nucleus of immune cells. CD4 ${ }^{+}$T-cell line CEM cells were fractionated into the cytosol and nucleus fractions using the same method as that for TZM-bl fractionation. As shown in Fig. 4c, a small amount of ENO1 located in the nucleus. To further confirm whether acute HIV-1 infection affects ENO1 nuclear translocation, TZMbl cells were infected with HIV-1 and fractionated. As a result, the same amount of ENO1 was detected in the nuclear fraction from noninfected or infected cells (Fig. 4d), indicating that ENO1 nuclear localization was unaffected by acute HIV-1 infection. These results suggest that the larger the amount of ENO1 present in the nucleus, the more HIV-1 integration is inhibited.

\section{ENO1 has bifunctional inhibitory activities on HIV-1 infection}

Finally, we clarified the bifunctional inhibitory activities of ENO1 in more detail. First, we prepared a high-levelENO1-packaging virus by cotransfection of HEK293 cells with pNL-CH and ENO1-V5 expression vector. As shown in Fig. 5a, lane 3, the high-level-ENO1-packaging virus showed significant decreases in its infectivity in normal TZM-bl cells. This finding is consistent with our previous findings [26]. Furthermore, as shown in Fig. 3b, the control virus showed significant decreases in its infectivity in the ENO1-overexpressing TZM-bl cells (Fig. 5a, lane 2). As expected, the high-level-ENO1-packaging virus showed a greater reduction in its infectivity in ENO1overexpressing TZM-bl cells (Fig. 5a, lane 4). Second, we prepared a low-level-ENO1-packaging virus from culture supernatants of ENO1-specific-siRNA-treated CEM/ LAV-1 cells. As shown in Fig. 5b, lanes 1 and 2, the control WT virus showed about $60 \%$ reduction in its infectivity in ENO1-overexpressing TZM-bl cells. In contrast, the low-level-ENO1-packaging virus also showed about $60 \%$ reduction in infectivity in the ENO1-overexpressing TZM-bl cells (Fig. 5b, lanes 3 and 4). These findings indicate that ENO1 in viral producer and target cells has bifunctional inhibitory activities on HIV-1 replication. 
a

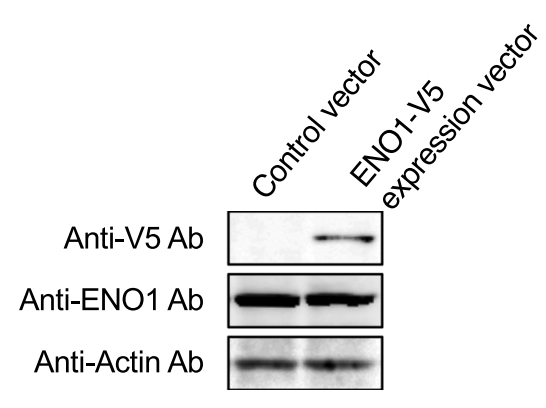

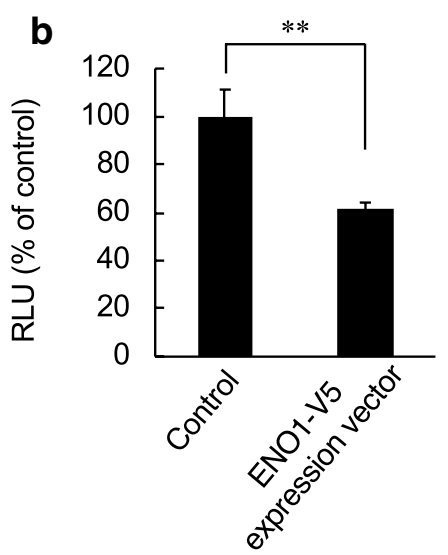

C

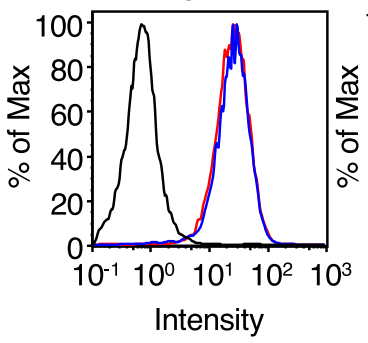

CD4

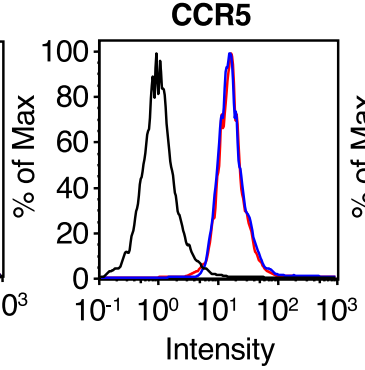

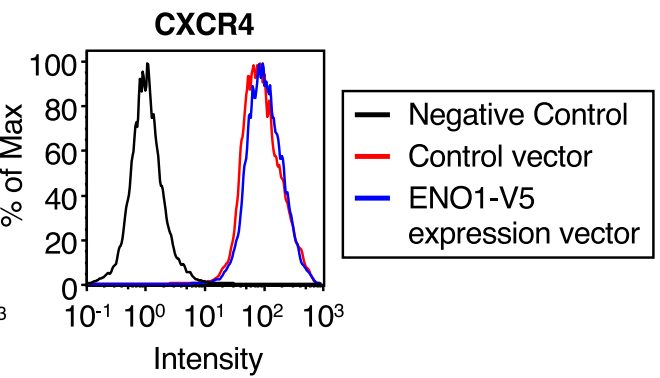

d

R/gag products

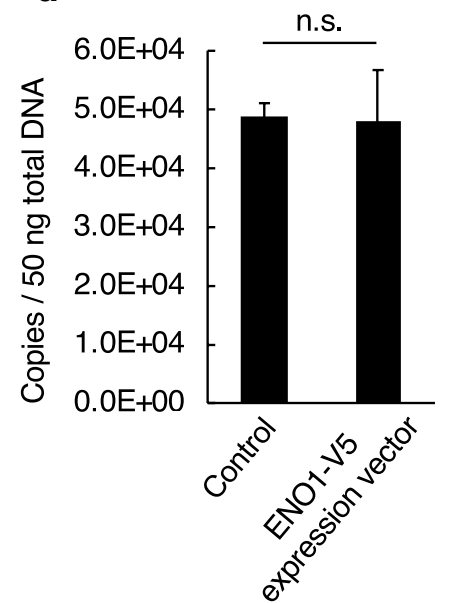

e

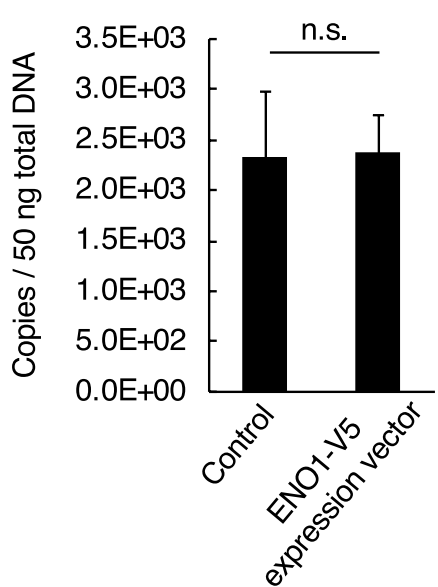

f

Alu-gag products

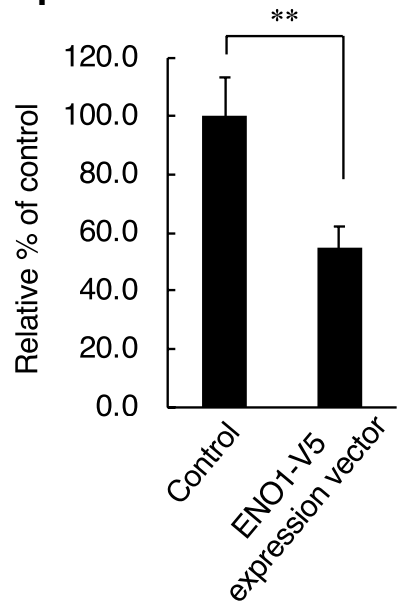

Fig. 3 Effects of ENO1 overexpression in HIV-1 target cells on postentry step. a Confirmation of ENO1-V5 expression in TZM-bl cells. Endogenous ENO1 was detected with an anti-ENO1 antibody and exogenous ENO1 (ENO1-V5) was detected with an anti-V5 antibody. b HIV-1 LAV-1 infectivity in ENO1-V5 expression vector-treated TZM-bl cells. The infectivity was examined at 2 days postinfection with HIV- LAV-1 $_{\text {and }}$ assessed on the basis of the luciferase activity in lysates of vector-treated TZM-bl cells. The infectivity in the control-vector-treated TZM-bl cells was set as 100\%. c Flow cytometry monitoring of HIV-1 receptor CD4 and coreceptors CCR5 and CXCR4. Cells stained with the isotype control antibody are indicated as negative control (black line). The expression levels of CD4, CCR5 and CXCR4 in control-vector- (red line) or ENO1-V5-expression vector (blue line)-treated TZM-bl cells are shown. Each protein was detected on the infection day. $\mathbf{d}$ Effect of ENO1-V5 expression in TZM-bl cells on viral reverse transcription. The amount of R/gag products of viral reverse transcription was determined by quantitative real-time PCR analysis. e Effect of ENO1-V5 expression in TZM-bl cells on viral cDNA nuclear import. The amount of 2-LTR circle products was determined by quantitative real-time PCR analysis. $\mathbf{f}$ Integration efficiency of HIV-1 LAV-1 $_{1}$ in either control- or ENO1-V5-expression vector-treated TZM-bl cells. Relative amount of Alu-gag products was determined by nested-PCR. The integration efficiency in the control-vector-treated TZM-bl cells was set as 100\%. Data are mean values \pm SE from triplicate tests. The significance of difference (Student's t-test) is indicated as follows: ${ }^{*}, p<0.01 ;{ }^{*}, p<0.05 ;$, n.s., not significant 
a

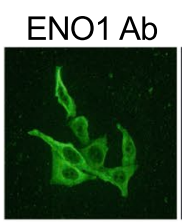

Endogenous ENO1

Anti-

Isotype

control
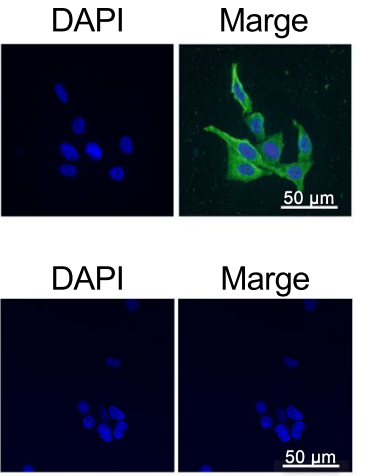

Exogenous ENO1
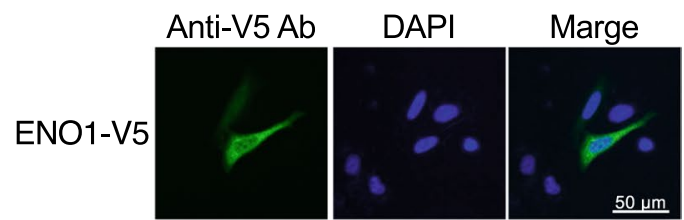

Isotype control
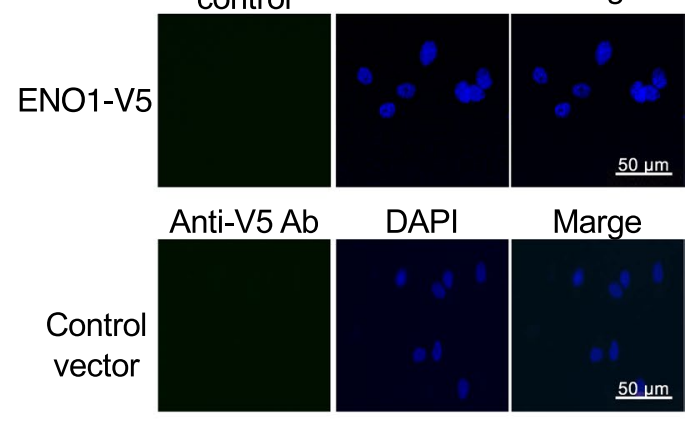

b

C

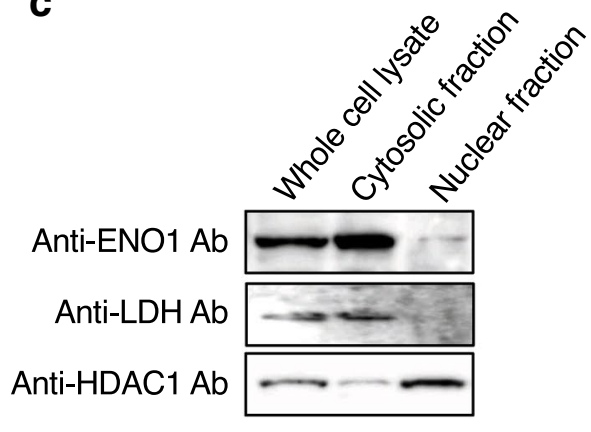

d

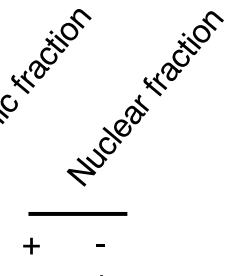

ENO1-V5-expression vector

Anti-ENO1 Ab

Anti-LDH Ab

Anti-HDAC1 Ab
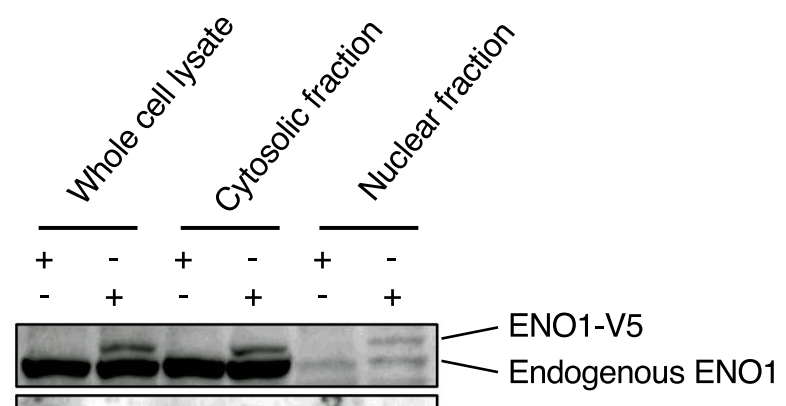

\section{$-$}

$-m+n-m$

\section{Endogenous ENO1}

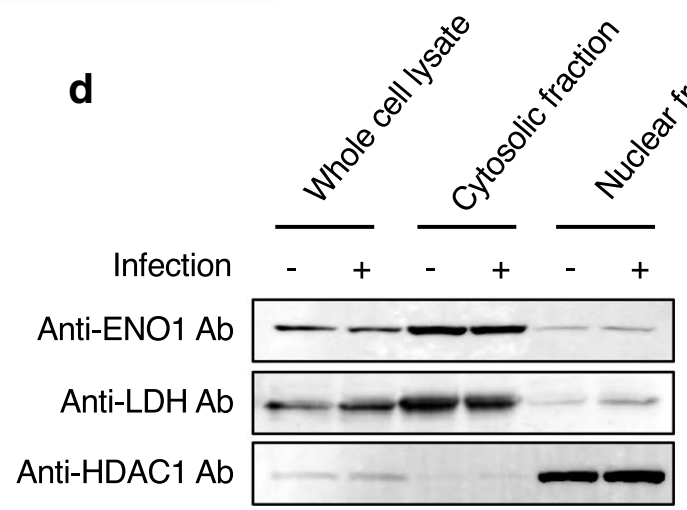

Fig. 4 ENO1 is present in the nucleus of the target cell. a Observation of endogenous ENO1 and ENO1-V5 by fluorescence microscopy. Endogenous ENO1 (left top panel) and ENO1-V5 (right top panel) were visualized by staining with the anti-ENO1 antibody and anti-V5 antibody, respectively. Nuclei were visualized by DAPI staining (middle column). The ENO1 or ENO1-V5 signal and DAPI signal are shown as merged (right column). In the right lower panels, cells transfected with the control vector were also analyzed. Small amounts of ENO1 and ENO1-V5 colocalized in the nucleus. A representative image from three independent experiments is shown. Subcellular fractionation of $\mathbf{b}$ TZM-bl cells, c CEM cells and d HIV-1 LAV-1-infected TZM-bl cells. b-d Endogenous ENO1 was detected by staining with the anti-ENO1 antibody and ENO1-V5 was detected by staining with the anti-V5 antibody. LDH and HDAC1 were detected as the cytosolic and nuclear markers, respectively. Fractionation was performed from the same cell number and then the amount loaded to each lane was calculated by the BCA method 


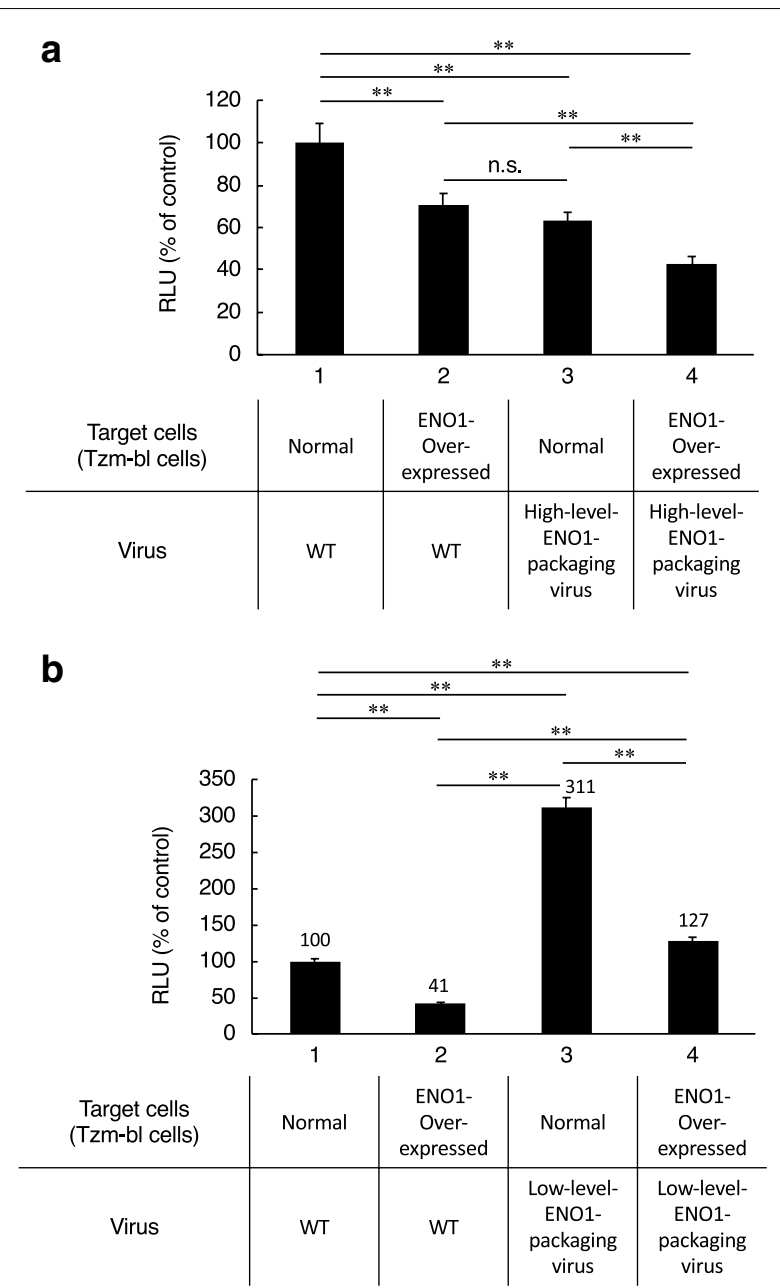

Fig. 5 ENO1 in viral producer and viral target cells has bifunctional inhibitory activities against HIV-1 replication. a Infection assay to confirm bifunctional inhibitory activity of ENO1. A high-level-ENO1-packaging virus derived from pNL-CH-transfected HEK293 cells and ENO1-overexpressing TZM-bl cells were prepared with an ENO1-V5 expression vector. The bifunctional inhibitory activity of ENO1 was examined by infecting target cells with different ENO1 expression levels using viruses with different amounts of ENO1 incorporated into their particles. $\mathbf{b}$ Infection assay to confirm bifunctional inhibitory activity of ENO1. A low-level-ENO1-packaging virus derived from CEM/LAV-1 cells treated with ENO1-specific siRNA and ENO1-overexpressing TZM-bl cells were prepared. The value in the control experiment (target cells and producer cells are shown as normal or WT) was set as 100\%. Data are mean values \pm SE from triplicate tests. The significance of difference (Nonrepeated measures ANOVA and Dunnett's test versus control) is indicated as follows: **, $p<0.01$; n.S., not significant

\section{Discussion}

Glycolytic enzymes have diverse functions as moonlighting proteins, and also regulate various infectious viruses. For example, GAPDH regulates hepatitis A virus, hepatitis $C$ virus and human parainfluenza virus infection and
ENO1 regulates Sendai virus and dengue virus infection [19-23]. In addition, we previously focused on virionassociated GAPDH and ENO1, and found that they independently prevent HIV-1 replication in HIV-1 producer cells [24-26]. Here, we examined whether GAPDH and ENO1 in viral target cells are involved in HIV-1 replication and found that GAPDH in the viral target cells is not involved in HIV-1 replication, whereas ENO1 in the viral target cells prevents HIV-1 replication by a mechanism different from that of ENO1 in viral producer cells. This is the first result showing that ENO1 inhibits viral replication even in HIV target cells.

It has not been clarified whether ENO1 in viral target cells is involved in HIV-1 replication. Here we showed that ENO1 in viral target cells has the capacity to prevent HIV-1 integration. We previously demonstrated that ENO1 was packaged into HIV-1 particles and the packaging of ENO1 suppresses the early stage of viral reverse transcription without inhibiting the packaging of cellular tRNA $^{\text {Lys3 }}$ into viral particles [26]. Unexpectedly, we found that the overexpression of ENO1 in viral target cells did not affect the number of copies of early and late cDNA products of HIV-1 reverse transcription (Supplementary Fig. 2 in [26] and Fig. 3d). However, we found that nuclear ENO1 expression in the viral target cells prevented HIV-1 integration. The ENO1 gene can give rise to a short $37 \mathrm{kDa}$ nuclear isoform, also called myc promoter binding protein 1 (MBP-1), which suppresses the activity of the c-myc transcription factor in the nucleus and lacks the first 96 amino acids [28]. In this study, as shown in Fig. 4b, we found that the same long form of ENO1 as the $48 \mathrm{kDa}$ enolase present in the cytoplasmic fraction was translocated to the nucleus, but no short MBP-1 was observed. The presence of the nuclear localization signal of ENO1 has not been described for any human, parasite, or plant ENO1 that is efficiently transported to the nucleus for repression of gene transcription [28-30]. Mouveaux et al. demonstrated that ENO1 of Toxoplasma gondii (TgENO1) exclusively localized in the nucleus [31]. Since the expression of about $48 \mathrm{kDa} H A-$ tagged TgENO1 has been confirmed by western immunoblotting [31], it is expected that its $N$-terminal long form has migrated into the nucleus and has the capacity for binding to putative gene promoters to control gene expression during the intracellular proliferation of $T$. gondii [31]. Furthermore, Cho et al. showed that ENOblock, a chemical probe for elucidating the moonlighting functions of ENO1, induces the nuclear localization of the long form of $48 \mathrm{kDa}$ ENO1 [32]. It is generally considered that proteins with a molecular weight of about $40 \mathrm{kDa}$ or larger cannot pass through the nuclear pore for their shuttling from the cytoplasm into the nucleoplasm. Interestingly, Cho et al. demonstrated that ENOblock 
enhances the nuclear localization of ENO1, but the effect of ENOblock is suppressed by treatment with an O-GlcNAc transferase inhibitor, OSMI-1 [33]. Although $10 \mu \mathrm{M}$ ENOblock did not promote ENO1 nuclear translocation in TZM-bl cells, compounds with the same action as ENOblock may suppress HIV integration by promoting $48 \mathrm{kDa}$ ENO1 translocation into the nucleus.

In the integration process, the viral integrase forms a preintegration complex (PIC) with a reverse-transcribed viral cDNA and cellular essential cofactors such as LEDGF/p75 and cleavage and polyadenylation specificity factor subunit 6 (CPSF6) $[6-8,34]$. Nuclear entry of HIV-1 PIC through the nuclear pore complex is an essential step in establishing HIV-1 infection. Bejarano et al. demonstrated that HIV-1 nuclear import in macrophages is regulated by CPSF6-capsid interactions at the nuclear pore complex [35]. Although there are differences between macrophages and cell lines, the expression level of ENO1 in the viral target cells did not affect the number of copies of 2-LTR circles forms (Figs. 2d, $3 e)$, suggesting that ENO1 is not directly involved in the process of HIV-1 PIC nuclear translocation. Following nuclear import, HIV-1 PIC utilizes both CPSF6 and LEDGF/p75 to target integration into transcriptionally active genes [34-38]. Recently, Sowd et al. found that CPSF6 directs HIV-1 to transcriptionally active chromatin, where LEDGF/p75 predominantly directs the positions of integration within active genes [34]. To clarify how ENO1 prevents viral integration, we carried out a coimmunoprecipitation assay. As far as we investigated, the coimmunoprecipitation assay showed that ENO1 did not directly interact with HIV-1 integrase or LEDGF/p75 (data not shown). In addition, the yeast-two hybrid analysis demonstrated that ENO1 also did not interact with HIV-1 proteins such as capsid, reverse transcriptase and integrase (Supplementary Fig. 2 in [26]). Furthermore, an increased ENO1 expression level decreases the efficiency of viral integration, and a decreased ENO1 expression level increases it (Figs. 2e, 3f). These findings suggest that ENO1 does not inhibit viral integration by directly binding to integrase or LEDGF/p75 and may suppress the viral integration within active genes by its direct binding to nearby active genes, as demonstrated by Subramanian and Miller [28] that the amino acids between 96 and 236 amino acids of ENO1 are essential for DNA interaction.

Since viruses replicate in host cells, information about the cellular environment, such as metabolism, is important for understanding virus replication. Hegedus et al. reported that HIV-1 infection increases in glycolytic flux [39]. Glucose transporter 1 is used as the main glucose transporter in activated T-cells, and its surface expression level is upregulated in HIV-1-infected $\mathrm{CD} 4^{+} \mathrm{T}$ cells [40]. Furthermore, HIV-1 infection increases hexokinase expression level and activity [41]. These lines of evidence demonstrated that HIV-1 replication requires glycolysisdependent cellular conditions. Interestingly, Hegedus et al. demonstrated that HIV-1 produced from cells cultured in galactose-containing medium, in which energy biosynthesis depends on oxidative phosphorylation and not glycolysis, showed decreased infectivity [39]. These findings led us to hypothesize that if glycolytic flux increases due to HIV-1 infection, glycolytic enzymes such as ENO1 must be involved in glycolysis, and HIV-1 replication will not be inhibited by ENO1.

\section{Conclusions}

We conclude that ENO1 in viral target cells inhibits HIV-1 integration. Together with our previous report, we consider ENO1 has bifunctional inhibitory activity against HIV-1 replication. This study strongly supports the possibility that glycolytic enzymes act as moonlighting proteins to inhibit HIV-1 replication.

\section{Methods \\ Cell culture}

CEM cells of the a CD4 ${ }^{+} \mathrm{T}$ cell line and CEM/LAV-1 cells of the chronically HIV-1 $\mathrm{LAV}-1$-infected T-cell line were maintained at $37{ }^{\circ} \mathrm{C}$ in RPMI-1640 supplemented with $10 \%$ fetal calf serum containing $100 \mathrm{IU} / \mathrm{ml}$ penicillin and $100 \mu \mathrm{g} / \mathrm{ml}$ streptomycin in $5 \% \mathrm{CO}_{2}$. TZM-bl cells and HEK293 cells were maintained in DMEM instead of RPMI-1640. TZM-bl cells were obtained from the NIH AIDS Research and Reference Reagent Program.

\section{Transfection}

Cells were transfected with $100 \mathrm{nM}$ validated commercially available Silencer ${ }^{\mathrm{TM}}$ GAPDH siRNA (Catalog \#:AM4605, Thermo Fisher Scientific Inc.) to suppress GAPDH expression and with $100 \mathrm{nM}$ validated commercially available Stealth ENO1 siRNA (Catalog \#:HSS103243, Thermo Fisher Scientific Inc.) to suppress ENO1 expression using the $\mathrm{Neon}^{\mathrm{TM}}$ transfection system (Thermo Fisher Scientific Inc.). To overexpress ENO1, cells were transfected with previously prepared pcDNA $^{\mathrm{TM}}$ 3.1D-ENO1-V5-His-TOPO ${ }^{\circledR}$ (Thermo Fisher Scientific Inc.; ENO1-V5 expression vector) using Lipofectamine $^{\circledR}$ LTX reagent and Plus ${ }^{\mathrm{TM}}$ Reagent (Thermo Fisher Scientific Inc.). To prepare the untagged ENO1 expression vector, the coding region of human ENO1 was cloned into pEBMulti-Neo (Wako Pure Chemical Industries, Ltd.). All experiments were performed in accordance with manufacturer's instructions. The expression level of each protein was determined by western immunoblotting using anti-GAPDH antibody (Merck KGaA), anti-ENO1 antibody (Santa Cruz Biotechnology, Inc.), anti-V5 antibody (Thermo Fisher Scientific Inc.), 
anti-actin antibody (Wako Pure Chemical industries, Ltd.) or HIV-1-positive plasma (a gift from Dr. Matsushita, Kumamoto University). The cytotoxicity induced by siRNA treatment was evaluated by trypan blue dye exclusion assay [24].

\section{Viruses}

Infectious HIV-1 $1_{\text {LAV-1 }}$ stocks were prepared from culture supernatants of CEM/LAV-1 cells. The low-level-ENO1packaging virus stocks were prepared from culture supernatants of ENO1-specific siRNA-treated CEM/LAV-1 cells, as previously described [26]. On the other hand, the high-level-ENO1-packaging virus stocks were prepared by cotransfection of HEK293 cells with the pNL-CH and ENO1-V5 expression vector, as previously described [26]. The release of each virus was directly monitored by $\mathrm{p} 24$ ELISA (ZeptoMetrix Corporation) as the amount of the CA protein in cell culture supernatants.

\section{Assessment of entry and postentry events}

The expressions of CD4, CCR5 and CXCR4 at the cell surface were confirmed at 1 day posttransfection by flow cytometry analysis using anti-CD4 antibody (Biolegend Inc.), anti-CCR5 antibody and anti-CXCR4 antibody (R\&D systems), respectively. The viral entry efficiency was determined by p24 ELISA (ZeptoMetrix Corporation) as the amount of the CA protein in the cytosolic fraction, as previously described [27]. Briefly, viral target cells were infected with each virus at $4{ }^{\circ} \mathrm{C}$ for $30 \mathrm{~min}$, and then incubated further for $4 \mathrm{~h}$ at $37{ }^{\circ} \mathrm{C}$. The cells were treated with $0.25 \%$ trypsin and then the cell cytosolic fraction was collected by two-step centrifugation (1st; $1000 \times g$, 2nd; $195,480 \times g$ ) after homogenization in swelling buffer $[10 \mathrm{mM}$ Tri- $\mathrm{HCl}(\mathrm{pH} 8.0), 10 \mathrm{mM} \mathrm{KCl}$, $1 \mathrm{mM}$ EDTA]. De novo synthesized viral cDNA products were measured by quantitative real-time PCR analysis in accordance with a previous methods [24, 26, 42]. To measure the amounts of R/gag, 2-LTR circle and Alu-gag products, DNA extracted from infected TZM-bl cells was amplified using the following primers. R/gag products: M667 (5'-GGCTAACTAGGGAACCCACTG-3') and M661 (5'-CCTGCGTCGAGAGAGCTCCTCTGG-3'); 2-LTR circle products: 2-LTR sense (5'-GAGATCCCT CAGACCCTTTTAG-3') and 2-LTR antisense (5'-GTC AGTCG- ATATCTGATCCCTG-3'); Alu-gag products: Alu-specific primer (5'-TCCCAGCTACTCGGGAGG CT- GAGG-3') and M661. To determine viral integration efficiency, Alu-gag products were further amplified using M667 and AA55 (5'-CTGCTAGAGATTTTCCACACTGAC-3'). Infectivity was determined by measuring the luciferase activity in cell lysates, which were prepared by a previously described method [20]. Viral infection was performed $24 \mathrm{~h}$ after transfection.

\section{Fluorescence microscopy and cell fractionation}

TZM-bl cells were seeded on an 8-well chambered Nunc $^{\text {TM }}$ Lab-Tek $^{\text {TM }}$ II Chamber Slide ${ }^{\text {TM }}$ system. At $24 \mathrm{~h}$ posttransfection, the cells were fixed with $1 \%$ paraformaldehyde, as previously described [43]. After that, endogenous ENO1 was detected with an anti-ENO1 antibody (Santa Cruz Biotechnology, Inc.) and exogenous ENO1 (ENO1-V5) was detected with an anti-V5 ENO1 antibody (Thermo Fisher Scientific Inc.). Normal Goat IgG control (Wako Pure Chemical industries, Ltd.) and mouse IgG2a (isotype control) (MEDICAL \& BIOLOGICAL LABORATORIES CO., LTD.) were used as negative control. Cells were fractionated using Subcellular Protein Fractionation Kit for Cultured Cells (Thermo Fisher Scientific Inc.) in accordance with the manufacturer's instructions. To fractionate cells after infection, the cells were seeded on a 6-well plate and incubated with HIV-1 $1_{\mathrm{LAV}-1}$ and $20 \mu \mathrm{g} / \mathrm{ml}$ DEAE dextran for $2 \mathrm{~h}$ and then cultured with cell growth medium for $48 \mathrm{~h}$.

\section{Supplementary information}

Supplementary information accompanies this paper at https://doi. org/10.1186/s12977-020-00539-9.

Additional file 1: Figure S1. Effects of untagged ENO1 in HIV-1 target cells.

\section{Abbreviations}

GAPDH: Glyceraldehyde 3-phosphate dehydrogenase; ENO1: Alpha-enolase; HIV-1: Human immunodeficiency virus type 1; LEDGF: Lens epithelium-derived growth factor; 2-LTR: Two-long terminal repeat; LDH: Lactate dehydrogenase; HDAC1: Histone deacetylase 1; MBP-1: Myc promoter binding protein 1; TgENO1: ENO1 of Toxoplasma gondii; PIC: Preintegration complex; CPSF6: Cleavage and polyadenylation specificity factor subunit 6 .

\section{Acknowledgements}

We thank Dr. R. Swanstrom (Linebarger Comprehensive Cancer Center, University of North Carolina at Chapel Hill) for providing pNL-CH and helpful discussions. We also thank Dr. Shuzo Matsushita (Joint Research Center for Human Retrovirus Infection, Kumamoto University, Kumamoto, Japan) for providing the HIV-1-positive plasma. TZM-bl cells were obtained from the AIDS Research and Reference Reagent Program, Division of AIDS, NIAID, NIH.

\section{Authors' contributions}

NK and SM conceptualized and designed the study; NK, KY, NI, CK, TA, NT, and SM performed the study and analyzed the data. NK, NI, and CK performed the siRNA experiment. NK and KY performed the overexpression experiment. NK, KY, TA, NT, and SM performed additional experiments. NK and SM wrote and critically read the paper. All the authors reviewed the manuscript. All authors read and approved the final manuscript.

\section{Funding}

This work was supported by JSPS KAKENHI Grant Numbers JP15H04659, JP16K18922 and JP19K16674.

Availability of data and materials

All data generated or analyzed during this study are included in this published article. 


\section{Ethics approval and consent to participate}

HIV-1-positive plasma was obtained from HIV-1-infected donors with informed consent by Dr. Shuzo Matsushita (Joint Research Center for Human Retrovirus Infection, Kumamoto University).

\section{Consent for publication}

Not applicable.

\section{Competing interests}

The authors declare that they have no competing interests.

\author{
Author details \\ ${ }^{1}$ Department of Environmental and Molecular Health Sciences, Faculty \\ of Medical and Pharmaceutical Sciences, Kumamoto University, Kuma- \\ moto 862-0973, Japan. ${ }^{2}$ Kumamoto Innovative Development Organization, \\ Kumamoto University, Kumamoto 860-8555, Japan.
}

Received: 22 November 2019 Accepted: 4 September 2020 Published online: 11 September 2020

\section{References}

1. Feng Y, Broder CC, Kennedy PE, Berger EA. HIV-1 entry cofactor: functional cDNA cloning of a seven-transmembrane, $G$ protein-coupled receptor. Science. 1996;272:872-7.

2. Cocchi F, Devico AL, Garzinodemo A, Arya SK, Gallo RC, Lusso P. Identification of Rantes, Mip-1-Alpha, and Mip-1-beta as the major Hiv-suppressive factors produced by Cd8(+) T-cells. Science. 1995;270:1811-5.

3. Deng HK, Liu R, Ellmeier W, Choe S, Unutmaz D, Burkhart M, DiMarzio P, Marmon S, Sutton RE, Hill CM, Davis CB, Peiper SC, Schall TJ, Littman DR, Landau NR. Identification of a major co-receptor for primary isolates of HIV-1. Nature. 1996:381:661-6.

4. Dragic T, Litwin V, Allaway GP, Martin SR, Huang YX, Nagashima KA, Cayanan C, Maddon PJ, Koup RA, Moore JP, Paxton WA. HIV-1 entry into CD4(+) cells is mediated by the chemokine receptor CC-CKR-5. Nature. 1996;381:667-73.

5. Alkhatib G, Combadiere C, Broder CC, Feng Y, Kennedy PE, Murphy PM, Berger EA. CC CKR5: a RANTES, MIP-1alpha, MIP-1 beta receptor as a fusion cofactor for macrophage-tropic HIV-1. Science. 1996;272:1955-8.

6. Cherepanov P, Maertens G, Proost P, Devreese B, Van Beeumen J, Engelborghs Y, De Clercq E, Debyser Z. HIV-1 integrase forms stable tetramers and associates with LEDGF/p75 protein in human cells. J Biol Chem. 2003:278:372-81.

7. Maertens G, Cherepanov P, Pluymers W, Busschots K, De Clercq E, Debyser Z, Engelborghs Y. LEDGF/p75 is essential for nuclear and chromosomal targeting of HIV-1 integrase in human cells. J Biol Chem. 2003;278:33528-39.

8. Llano M, Saenz DT, Meehan A, Wongthida P, Peretz M, Walker WH, Teo W, Poeschla EM. An essential role for LEDGF/p75 in HIV integration. Science. 2006:314:461-4.

9. Sheehy AM, Gaddis NC, Choi JD, Malim MH. Isolation of a human gene that inhibits HIV-1 infection and is suppressed by the viral Vif protein. Nature. 2002;418:646-50.

10. Mangeat B, Turelli P, Caron G, Friedli M, Perrin L, Trono D. Broad antiretroviral defence by human $A P O B E C 3 G$ through lethal editing of nascent reverse transcripts. Nature. 2003:424:99-103.

11. Laguette N, Sobhian B, Casartelli N, Ringeard M, Chable-Bessia C, Ségéral E, Yatim A, Emiliani S, Schwartz O, Benkirane M. SAMHD1 is the dendriticand myeloid-cell-specific HIV-1 restriction factor counteracted by Vpx. Nature. 2011:474:654-7.

12. Hrecka K, Hao C, Gierszewska M, Swanson SK, Kesik-Brodacka M, Srivastava S, Florens L, Washburn MP, Skowronski J. Vpx relieves inhibition of HIV-1 infection of macrophages mediated by the SAMHD1 protein. Nature. 2011;474:658-61.

13. Jeffery CJ. Moonlighting proteins. Trends Biochem Sci. 1999;24:8-11

14. Jeffery CJ. Why study moonlighting proteins? Front Genet. 2015;6:211.

15. Castello A, Hentze MW, Preiss T. Metabolic enzymes enjoying new partnerships as RNA-binding proteins. Trends Endocrinol Metab. 2015:26:746-57.
16. Ishitani R, Chuang DM. Glyceraldehyde-3-phosphate dehydrogenase antisense oligodeoxynucleotides protect against cytosine arabinonucleoside-induced apoptosis in cultured cerebellar neurons. Proc Natl Acad Sci USA. 1996;93:9937-41

17. Sirover MA. On the functional diversity of glyceraldehyde-3-phosphate dehydrogenase: biochemical mechanisms and regulatory control. Biochim Biophys Acta. 2011;1810:741-51.

18. Volker KW, Reinitz CA, Knull HR. Glycolytic enzymes and assembly of microtubule networks. Comp Biochem Physiol B Biochem Mol Biol. 1995;112:503-14

19. Schultz DE, Hardin CC, Lemon SM. Specific interaction of glyceraldehyde 3-phosphate dehydrogenase with the 5'-nontranslated RNA of hepatitis A virus. J Biol Chem. 1996;271:14134-42.

20. Duclos-Vallee JC, Capel F, Mabit H, Petit MA. Phosphorylation of the hepatitis $B$ virus core protein by glyceraldehyde-3-phosphate dehydrogenase protein kinase activity. J Gen Virol. 1998;79:1665-700.

21. Petrik J, Parker H, Alexander GJ. Human hepatic glyceraldehyde-3-phosphate dehydrogenase binds to the poly $(U)$ tract of the 3 ' non-coding region of hepatitis C virus genomic RNA. J Gen Virol. 1999;80:3109-13.

22. Munoz Mde L, Limon-Camacho G, Tovar R, Diaz-Badillo A, MendozaHernandez G, Black WC 4th. Proteomic identification of dengue virus binding proteins in Aedes aegypti mosquitoes and Aedes albopictus cells. Biomed Res Int. 2013;2013:875958.

23. Ogino T, Yamadera T, Nonaka T, Imajoh-Ohmi S, Mizumoto K. Enolase, a cellular glycolytic enzyme, is required for efficient transcription of Sendai virus genome. Biochem Biophys Res Commun. 2001;285:447-55.

24. Kishimoto N, Onitsuka A, Kido K, Takamune N, Shoji S, Misumi S. Glyceraldehyde 3-phosphate dehydrogenase negatively regulates human immuno-deficiency virus type 1 infection. Retrovirology. 2012:9:107.

25. Kishimoto N, Onitsuka-Kishimoto A, Iga N, Takamune N, Shoji S, Misumi $\mathrm{S}$. The C-terminal domain of glyceraldehyde 3-phosphate dehydrogenase plays an important role in suppression of tRNALys 3 packaging into human immunodeficiency virus type-1 particles. Biochem Biophys Rep. 2016:8:325-32.

26. Kishimoto N, Iga N, Yamamoto K, Takamune N, Misumi S. Virion-incorporated alpha-enolase suppresses the early stage of HIV-1 reverse transcription. Biochem Biophys Res Commun. 2017;484:278-84.

27. Marechal V, Clavel F, Heard JM, Schwartz O. Cytosolic Gag p24 as an index of productive entry of human immunodeficiency virus type 1. J Virol. 1998;72:2208-12.

28. Subramanian A, Miller DM. Structural analysis of alpha-enolase. Mapping the functional domains involved in down-regulation of the c-myc protooncogene. J Biol Chem. 2000;275(8):5958-65.

29. Feo S, Arcuri D, Piddini E, Passantino R, Giallongo A. ENO1 gene product binds to the c-myc promoter and acts as a transcriptional repressor: relationship with Myc promoter-binding protein 1 (MBP-1). FEBS Lett. 2000:473(1):47-52.

30. Lee H, Guo Y, Ohta M, Xiong L, Stevenson B, Zhu JK. LOS2, a genetic locus required for cold-responsive gene transcription encodes a bi-functional enolase. EMBO J. 2002;21(11):2692-702.

31. Mouveaux T, Oria G, Werkmeister E, Slomianny C, Fox BA, Bzik DJ, Tomavo S. Nuclear glycolytic enzyme enolase of Toxoplasma gondii functions as atranscriptional regulator. PLoS ONE. 2014;9(8):e105820.

32. Cho H, Um J, Lee JH, Kim WH, Kang WS, Kim SH, Ha HH, Kim YC, Ahn YK, Jung DW, Williams DR. ENOblock, a unique small molecule inhibitor of the non-glycolytic functions of enolase, alleviates the symptoms of type 2 diabetes. Sci Rep. 2017;7:44186.

33. Cho H, Lee JH, Um J, Kim S, Kim Y, Kim WH, Kim YS, Pagire HS, Ahn JH, Ahn Y, Chang YT, Jung DW, Williams DR. ENOblock inhibits the pathology of diet-induced obesity. Sci Rep. 2019:9(1):493.

34. Sowd GA, Serrao E, Wang H, Wang W, Fadel HJ, Poeschla EM, Engelman AN. A critical role for alternative polyadenylation factor CPSF6 in targeting HIV-1 integration to transcriptionally active chromatin. Proc Natl Acad Sci USA. 2016:113(8):E1054-E10631063.

35. Bejarano DA, Peng K, Laketa V, Börner K, Jost KL, Lucic B, Glass B, Lusic M, Müller B, Kräusslich HG. HIV-1 nuclear import in macrophages is regulated by CPSF6-capsid interactions at the nuclear pore complex. Elife. 2019. https://doi.org/10.7554/eLife.41800.

36. Ciuffi A, Llano M, Poeschla E, Hoffmann C, Leipzig J, Shinn P, Ecker JR, Bushman F. A role for LEDGF/p75 in targeting HIV DNA integration. Nat Med. 2005:11(12):1287-9. 
37. Shun MC, Raghavendra NK, Vandegraaff N, Daigle JE, Hughes S, Kellam P, Cherepanov P, Engelman A. LEDGF/p75 functions downstream from preintegration complex formation to effect gene-specific HIV-1 integration. Genes Dev. 2007;21(14):1767-78.

38. Marshall HM, Ronen K, Berry C, Llano M, Sutherland H, Saenz D, Bickmore W, Poeschla E, Bushman FD. Role of PSIP1/LEDGF/p75 in lentiviral infectivity and integration targeting. PLOS ONE. 2007;2(12):e1340.

39. Hegedus A, Kavanagh Williamson M, Huthoff H. HIV-1 pathogenicity and virion production are dependent on the metabolic phenotype of activated CD4+ T cells. Retrovirology. 2014;11:98.

40. Palmer CS, Ostrowski M, Gouillou M, Tsai L, Yu D, Zhou J, Henstridge DC, Maisa A, Hearps AC, Lewin SR, Landay A, Jaworowski A, McCune $J M$, Crowe SM. Increased glucose metabolic activity is associated with CD4+ T-cell activation and depletion during chronic HIV infection. AIDS 2014;28:297-309.

41. Kavanagh Williamson M, Coombes N, Juszczak F, Athanasopoulos M, Khan MB, Eykyn TR, Srenathan U, Taams LS, Dias Zeidler J, Da Poian AT,
Huthoff $\mathrm{H}$. Upregulation of glucose uptake and hexokinase activity of primary human CD4+ T cells in response to infection with HIV-1. Viruses. 2018;10(3):114

42. Ikeda T, Nishitsuji H, Zhou X, Nara N, Ohashi T, Kannagi M, Masuda T. Evaluation of the functional involvement of human immunodeficiency virus type 1 integrase in nuclear import of viral cDNA during acute infection. J Virol. 2004;78:11563-73.

43. Ogawa M, Takemoto Y, Sumi S, Inoue D, Kishimoto N, Takamune N, Shoji S, Suzu S, Misumi S. ATP generation in a host cell in early-phase infection is increased by upregulation of cytochrome c oxidase activity via the $\mathrm{p} 2$ peptide from human immunodeficiency virus type 1 Gag. Retrovirology. 2015;12:97.

\section{Publisher's Note}

Springer Nature remains neutral with regard to jurisdictional claims in published maps and institutional affiliations.
Ready to submit your research? Choose BMC and benefit from:

- fast, convenient online submission

- thorough peer review by experienced researchers in your field

- rapid publication on acceptance

- support for research data, including large and complex data types

- gold Open Access which fosters wider collaboration and increased citations

- maximum visibility for your research: over $100 \mathrm{M}$ website views per year

At BMC, research is always in progress.

Learn more biomedcentral.com/submissions 